\title{
The Computer Experimental Teaching Platform Based on Cloud Computing Technology
}

\author{
Jing $\mathrm{Cai}^{1, \mathrm{a}}$ \\ ${ }^{1}$ Nanchang Institute of Science and Technology, 330108 \\ acaijingjx@126.com
}

Keywords: cloud computing; computer; experimental teaching platform

\begin{abstract}
With the all-sided development of the society, the integral application of cloud computing technology in computer teaching platform is critical. It can not only improve the teaching efficiency generally, but also improve the computing experimental teaching platform in all-around way. This paper mainly analyzes the application of cloud computing technology to the computer experimental teaching platform, and puts forward the corresponding optimizing measures.

In the process of overall application of cloud computing, it is necessary to combine computer experimental teaching platform to improve its proficiency. However, there are still many teaching problems in many sides in real teaching process, in order to cloud computing technology can be applied comprehensively, it is necessary to optimize the experimental teaching platform in all -around way.
\end{abstract}

\section{The Construction of computing teaching platform based on the cloud computing technology \\ Platform architecture}

Cloud computing is a style of computing in which dynamically scalable and often virtualized resources are provided as a service over the Internet. Cloud computing provides Cloud computing is available and convenient, and provide the corresponding network access, and then enter a shared pool of configurable computing resources that can be rapidly provided. You just need do some management effort next, because it has the characteristics of dynamic resources disposition, automatic services and taking the network as the center, the service being measurable etc.

Service and management are the two parts of computer experimental teaching platform architecture of cloud computing. As for service aspect, it provides various services of cloud computing, including IaaS(Infrastructure-as-a- Service), PaaS(Platform-as-a- Service and SaaS(Software-as-a- Service) . Lass is to provide customers with various computing resources a computer through Internet, such as storage, network link, etc. Various software operation and deployment can be on the basis of this, including the operating system and applying programs. PaaS is as a service of software development, and then it is given to the user in the SaaS model. The platform is divided into operating systems, running environment of programming languages, database and Web server, the user can run their applications on the platform. Moreover, users can not operate the management operation and bottom control operation, they can just control their own. SaaS is a software model provided by Internet, users don't need to buy by their own. Actually, from the user's point of view, they are all independent, the services they provided are completely different, and users are also different, but from the technical layer, the three layers have a certain relationship.

As for management aspect, it is mainly the cloud management layer, which has the function of ensuring the cloud management can function safely, stably and proficiently.

\section{The Implementation of Platform}

When you start the client for the first time, there will be system data sent automatically on the client's hard disk. The hard disk can store the user' data and the temporary files of system at the same time and then form a high-speed cache. If users need more data when they are using their 
computer, the server can directly send corresponding data from the hard disk to users, thus, the client can mark and record both the data sent by the server and the data created by users to form a better cache. So each time you start the client, a small amount of I/O reading and writing will be transmitted. Starting data is the combination of partial data and cache. Congress can ensure user experience is completely consistent with stand-alone mode. Due to managing image files of virtual desktop serve is based on the computer experimental system, when users start their computers, the server will assign private application system and desktop. The assignment is based on the different accounts logged in after the computer is booted up. So that it can recommend personalized demand for each class and person.

\section{The Accomplishment of the Function of Computer Teaching Experimental Platform Based on Cloud Computing.}

\section{Centralized deployment can reduce the cost of the deployment and maintenance, and reduce the time of deployment.}

If we want to reduce the cost of installation and deployment, we can deploy them intensively, so that it only takes a few minutes to distribute any application in laboratory. We have to upgrade and maintain the critical application of the client in time. The upgrade of application also occurs on the server. It is unnecessary to install all the application on the client, and the required property is not too good.

\section{Practicability}

Making it more convenient for students to use computers and letting them involved into information age more quickly is the main purpose of information construction in school. However, the lack of fund slows the pace of it. At the same time, the computer experimental teaching platform alleviates the problem, and it is easier to operate. Cloud terminal has many advantages, not only there are no moving parts, also it can share resources, such as hardware and software resources of the server; teaching terminal can use the teaching software of the server terminal, etc. all these can prove that cloud terminal is more convenient to use.

\section{Low Cost}

When the laboratory is built up and we are purchasing computers, we must stick to the "enough purchase of computers at a time, top-level configuration" mode. The purpose of ding this is to reduce satisfy the need of teaching in the future years. With the development of the information technology and the increasing of experience, we need to learn how to reduce the cost of network teaching of school, including the original invest of hardware, the cost of purchasing applied software and system software, and the cost of installation and debugging of system software and hardware.

\section{Proficient management and easy maintenance}

“Cloud, Channel, Device” experimental platform conducts centralized management to computers. So the managers can pay a watchful eye to the usage of computer without needing to come in front the computers, and can manage and control the computers at any time. For example, the system maintenance in a large scale and the installation and upgrade of system software can be achieved by just one computer. There is no need that every computer has to be operating. Comparing to the method of the original card, the method adopted by "Cloud, Channel, Device" system can reduce more than half the time.

\section{Safety and Reliability}

As for the remote maintenance and diagnosis, computer experimental platform can conduct remote maintenance and diagnosis to the computers, however, as for the wrong operations, we need managers to maintain them, and this just need several people. Thus the time and cost of maintenance is reduced to a great extent. As for the aspect of fault tolerance, the old computer 
rooms in school are easier to go wrong, which cause the system unable to function properly, so if it is based on cloud computing, the network can be guaranteed to be normal. For the presence of these faults, such as, updating virus library and upgrading patches, this platform can guarantee the system will not go wrong, and decrease the chance of loss.

\section{Personalized service}

Computer experiment teaching platform based on cloud computing technology provides the conventional network boot mode, which provides the convenience of management. However, it can not work normally if there is something wrong. So the starting system by starting local operating system and using local cache which is provided by computer experiment teaching platform solve the above problems very well. Moreover, there are various ways according to the practical needs and different requirements of clients, including providing start reduction, reduction, reducing only the next time and so on a variety of ways of reduction.

\section{Summary}

The optimization of the computer experimental teaching platform based on cloud computing needs consider many factors. You need make a comprehensive change to the system, but also design the experimental function system of computers in order to realize all kinds of teaching function well, so that better teaching effect can be achieved.

\section{References}

[1] Yang Fengqing. A Study on the Cloud Computing Aided Teaching Platform Based on Zoho [J]. Software Tribune (Education). 2013(02)

[2] Sun Yiquan. The Design of "Cloud Computing” Simulation Experiment for Open Source Software [J]. Experimental Technology and Management. 2013(02)

[3] Guo Peiyuan, Fengbo. The Design and Implementation of Virtual Laboratory Based on Cloud Computing Platform][J]. Software. 2012(10)

[4] Jiangning, Liwen, Li Hongbing. A Study on the Cloud Computing Aided Teaching Platform Based on Hadoop[J]. Chinese Remote Education. 2012(09) 